\title{
El Aprendizaje Basado en Problemas (ABP) en Ciencias Sociales: resultados de una experiencia en estudiantes de postgrado en Chile
}

\author{
Carla Vidal Figueroa, Gustavo Castillo Rozas ${ }^{\mathrm{b}}$, Claudia Castillo Rozas ${ }^{\mathrm{c}}$ \\ ${ }^{a}$ Profesora Asistente, Departamento de Trabajo Social, Universidad de Concepción, Chile, \\ carvidal@udec.cl. 'Profesor Asistente, Departamento de Trabajo Social, Universidad de Concepción, \\ Chile, gucastillo@,udec.cl. ${ }^{c}$ Profesora Asistente, Departamento de Trabajo Social, Universidad de \\ Concepción, Chile, ccastill@udec.cl.
}

\section{Resumen}

El método ABP - Aprendizaje Basado en Problemas (Learning Based Problem) tiene como objetivo central el transformar la dinámica tradicional del trabajo con estudiantes en el aula, fomentando en los y las jóvenes la búsqueda de respuestas mediante acciones colaborativas con el grupo, que surgen de un escenario propuesto y que les ayuda a definir sus propios objetivos de aprendizaje durante el curso. El trabajo que realizan de forma autónoma les permite aportar con información novedosa el desarrollo de las sesiones grupales, convirtiéndose el $A B P$ en una herramienta que no se orienta a que los y las estudiantes resuelvan los problemas por si mismos, sino que busca utilizar los conocimientos que se generan en un entorno de reflexión y análisis grupal para aumentar el conocimiento y comprensión del mismo problema, pero con diferentes perspectivas. Sobre esta base, se utilizó la metodología en un módulo del Diplomado en Gestión de Políticas Sociales de la Universidad de Concepción, con estudiantes egresados de diferentes carreras. La experiencia se valoró como exitosa debido al alto grado de participación que se observó en los/las estudiantes y del compromiso por determinar la problemática que se estaba trabajando aportando con argumentos que surgían de la reflexión personal y posterior análisis grupal. El presente artículo pretende ilustrar esta experiencia y confirmar dado los resultados obtenidos, que el ABP es una herramienta válida, cuyos beneficios permiten desarrollar en los y las estudiantes diferentes habilidades basadas en las competencias, como generar un análisis crítico, el trabajo en equipo, y la investigación.

Palabras clave: aprendizaje basado en problemas, ABP, competencias, trabajo en equipo, análisis crítico 
El Aprendizaje Basado en Problemas (ABP) en Ciencias Sociales: resultados de una experiencia en estudiantes de postgrado en Chile

\section{Introducción}

Atendiendo a los desafíos que día a día presenta la enseñanza en la educación superior desde el punto de vista docente, es preciso aprender, desarrollar y evaluar nuevas metodologías que permitan acercar a los estudiantes a las temáticas que se abordan en el contexto universitario, sea este pregrado y/o postgrado. Dicho acercamiento, ha de responder además a una actualización en cuanto a técnicas de enseñanza que permitar situar al estudiante de aula en el centro de la dinámica que se experimenta clase a clase, delegando en su figura la responsabilidad del aprendizaje y por consiguiente, de la puesta en práctica de todos los elementos adquiridos en dichas instancias. No cabe duda el constante cuestionamiento que está presente en el quehacer del docente durante su vida académica ¿qué tan preparados están nuestros estudiantes al término de sus estudios para ejercer su profesión? Y siendo el entorno universitario tan diverso en cuanto el nivel socioeconómico de sus estudiantes ¿cómo equiparar el desarrollo de habilidades durante la etapa universitaria? ¿puede la experiencia universitaria reducir esta brecha?

Siguiendo esta lógica la Universidad de Concepción (Chile) elaboró un modelo educativo que se orienta en el desarrollo de competencias en las/os estudiantes, compuesto a su vez por cuatro macrocompetencias genéricas: pensamiento crítico, comunicación (verbal-no verbal/oral-escrita), emprendimiento $y$ trabajo en equipo interdisciplinario y responsabilidad social (Programa de Estudios sobre la Responsabilidad Social , 2013). Sobre esta base se concibe a la metodología de Aprendizaje Basado en Problemas (en adelante $\mathrm{ABP}$ ) como una forma de dar respuesta a dichas macrocompetencias, exponiendo en el presente artículo los resultados de una experiencia llevada a cabo en dos períodos, y que representa la aplicación de esta metodología en un grupo de estudiantes de postgrado de la Universidad de Concepción. Si bien es cierto y tal como se expondrá mas adelante, con esta metodología no se pretende reducir la brecha de desigualdad económica que existe en el contexto de la educación superior, si podemos apuntar a nivelar a sus estudiantes en cuanto al desarrollo de habilidades y competencias para enfrentar con éxito el futuro mundo laboral, ofreciendo una igualdad de condiciones al menos en ambos aspectos.

\section{Aprendizaje Basado en Problemas (ABP) como una herramienta efectiva}

El método de Aprendizaje Basado en Problemas (ABP), es definido por Barrows (1986) como un método de aprendizaje basado en el principio de usar problemas como punto de partida para la adquisición e integración de los nuevos conocimientos. Es una metodología que surge en los años 60's desde el área de la medicina en la Universidad de McMaster en Canadá, enfocada en lograr una mejor preparación de los estudiantes que pudiese complementar aquellos conocimientos que adquirían mediante la ensañanza tradicional en 
las aulas con aquellos adquiridos a través de la práctica profesional. El cambio se produjo cuando los profesionales fueron conscientes de la necesidad de que el alumnado adquiriera habilidades para la solución de problemas, para lo que era necesario desarrollar otras habilidades como la de adquirir información, sintetizarla, generar y probar ciertas hipótesis de acuerdo a la información recogida, denominando a este proceso como Razonamiento Hipotético Deductivo (Morales \& Landa, Aprendizaje Basado en Problemas , 2004), propuesta que se conoce actualmente como Aprendizaje Basado en Problemas (ABP).

Resulta factible pensar entonces, en adaptar este modelo a diversas áreas del conocimiento, más aún cuando el contexto académico exige replantear las estrategias y la dinámica que se utilizan en el aula apuntando hacia una mayor participación durante la sesión por parte de las/os estudiantes. Esto adquiere mayor relevancia cuando se piensa en las habilidades que deben desarrollar de cara a enfrentar el mundo laboral, mediante procesos que son cada vez más competitvos y que demanda de las/os egresados poner en práctica un conjunto de destrezas y habilidades que se debieron haber adquirido en su etapa universitaria. Por lo tanto, asumir la implementación de una metodología como el ABP, debería implicar un cambio conceptual hacia una visión constructivista del aprendizaje, según la cual la complejidad del proceso de enseñanza-aprendizaje y los diversos factores que se interrelacionan en él — como el currículo, los estudiantes, los docentes, la metodología, el clima de interacción y los procedimientos de evaluación-, conforman un sistema único en cada situación educativa (Morales, 2009, pág. 76).

Los nuevos tiempos demandan una innovación de las prácticas que se llevan a cabo en las salas de clase y un mayor protagonismo por parte de quienes reciben la formación, avogando por generar un rol más participativo y un compromiso con la temática que se está desarrollando, traspasando la responsabilidad en la adquisición del conocimiento a las/os estudiantes. En este sentido, Barrel (1999) destaca algunos de los beneficios del uso del $\mathrm{ABP}$ en el aula que pueden responder a lo que se plantea, como por ejemplo, que la indagación y reflexión sobre la práctica conducen a una comprensión más profunda, que existe un mayor aprendizaje cuando la información se usa de manera significativa y que en experimentos controlados, se incrementa el uso de estrategias para la resolución de problemas, por lo que se obtiene más información que en el caso de los estudiantes que reciben clases tradicionales.

Dicha metodología, apunta entonces a conseguir el éxito educativo, que de acuerdo a lo planteado por Barber y Mourshed (2008, pág. 14), dependerá de tres factores fundamentales: la calidad de las/os docentes, la mejora de los procesos de enseñanzaaprendizaje en el aula (instrucción) y la consecución de la mejora del nivel para todos los estudiantes. Por lo tanto, al utilizar esta metodología apuntamos a que el/la estudiante busque lo que necesita aprender para poder desarrollar el problema y por lo tanto incorpore mayor información que sirva de base para generar un debate y discutir con el resto del 
El Aprendizaje Basado en Problemas (ABP) en Ciencias Sociales: resultados de una experiencia en estudiantes de postgrado en Chile

grupo. Sobre la base de esta propuesta es que se plantea la metodología que se expone a continuación y que busca ser una alternativa en términos de enseñar, generar conocimiento y desarrollar determinadas habilidades en las/os estudiantes que facilite el ingreso al mercado laboral y los prepare para los desafíos que este presenta. Para ello, se ilustrará en los siguientes apartados una experiencia exitosa que se llevó a cabo en un módulo del Diplomado en Gestión de Políticas Sociales en los años 2015 y 2016, que confirma que el ABP es una herramienta válida, cuyos beneficios permiten desarrollar en las/os estudiantes el análisis crítico, el trabajo en equipo, y la investigación, todo dentro de la sala de clases.

\section{Metodología}

Los resultados de la experiencia que se exponen en este artículo, fue desarrollada en dos versiones del Diplomado en Gestión de Políticas Sociales perteneciente al Departamento de Ciencias Políticas y Administrativas de la Universidad de Concepción (Chile), específicamente en el módulo sobre Estados de Bienestar. En el año 2015 participaron 11 estudiantes y 16 estudiantes en el año 2016, quienes por medio del Aprendizaje Basado en Problemas (ABP) desarrollaron los contenidos del módulo. Este se configuró de la siguiente manera:

Objetivo del módulo. Conocer la estructura de bienestar presente en los países del contexto Europeo y Latinoamericano y cómo rige su aplicación a través de las políticas sociales.

Resultados de aprendizaje esperados. Durante dos sesiones (viernes y sábado) que en total suman 7 horas, los/as estudiantes deben ser capaces de: mostrar iniciativa y disposición al aprendizaje, desarrollar una actitud de respeto frente a la oponión de sus compañeros/as, emplear adecuadamente habilidades comunicativas que le hagan cuestionar en equipo la problemática abordada, identificar los aspectos de la noticia vinculada con el pilar del bienestar que le corresponde al grupo, fortalecer el trabajo investigativo y autónomo, el análisis crítico, y capacidad de trabajar en equipo, competencias propias de la estrategia ABP.

Metodología de trabajo. La actividad comienza enseñando a las/os estudiantes las principales caracterísitcas del ABP y los beneficios que genera su aplicación en el aula, instancia que se considera como una ventaja para la estrategia pues los estudiantes saben lo que se espera de ellos y los beneficios que conlleva aprender los contenidos de esta forma. El curso se divide en dos grupos a los cuales se les asigna una noticia vinculada a un modelo de bienestar y sobre el cual deben dilucidar la temática a trabajar en el módulo y durante las dos sesiones, debiendo preparar en la última sesión la información a incluir en un informe final. 
El rol del profesor-tutor. Desempeñar la función de guía y facilitador durante el trabajo del grupo. Se encargará de observar los aportes de cada uno de los integrantes, cuyo desempeño será evaluado a nivel grupal e individual al término del módulo. En ambas versiones se trabajó con dos docentes en el aula, cada uno a cargo de un grupo.

Evaluación de la sesión. La evaluación final del módulo se realiza en el aula y es de carácter grupal. Consiste en la exposición de los hallazgos encontrados por el grupo durante las sesiones de trabajo mediante plenario y con la entrega de un informe. Los/las estudiantes también son evaluados de forma individual, utilizando los siguientes instrumentos y rúbricas elaborados para la actividad, y basados en el trabajo realizado por Rivera, Bruna, Reinicke, Grez \& Santamaría (2014): escala de estimación conceptual por cada alumno/a (evaluación individual realizada por el tutor), pauta para co-evaluar el trabajo colaborativo (estudiantes evalúan a sus compañeros/as), pauta para autoevaluar el trabajo colaborativo (estudiantes evalúan su aportación al trabajo desarrollado).

Por último, y con el fin de obtener una retroalimentación de la actividad, los/as estudiantes responden una lista de cotejo en la que plasman sus apreciaciones respecto al trabajo desarrollado. Sobre este último instrumento es que se exponen los resultados a continuación.

\section{Resultados}

A continuación se presentan los resultados de la escala de estimación conceptual aplicada a cada una/o de las/os estudiantes del módulo, considerando su valoración en aspectos como la metodología, la dinámica, el aprendizaje, los instrumentos de evaluación utilizados y una evaluación general de la actividad.

Respecto a la metodología utilizada, la totalidad de las/os estudiantes reconoce que durante las dos sesiones se sintieron motivados a trabajar y desarrollar la temática asignada de forma independiente, favoreciendo su capacidad de análisis y estudio autónomo. Cabe destacar que el tiempo entre una sesión y la otra era escaso por lo cual las/os estudiantes realizaban un esfuerzo por investigar de forma independiente sobre el tema con el objetivo de llegar a la segunda sesión con material que pudieran utilizar para discutir con sus compañeras/os. 
El Aprendizaje Basado en Problemas (ABP) en Ciencias Sociales: resultados de una experiencia en estudiantes de postgrado en Chile

Tabla 1. Evaluación de la Metodología

\begin{tabular}{|l|c|c|c|c|}
\hline \multicolumn{1}{|c|}{ Aspecto que el/la estudiante evalúa } & \multicolumn{2}{c}{$2015(\%)$} & \multicolumn{2}{c|}{$2016(\%)$} \\
\hline $\begin{array}{l}\text { La metodología utilizada me motivó a estudiar } \\
\text { sobre la temática del módulo }\end{array}$ & 100 & -- & 100 & -- \\
\hline La metodología fomentó el estudio independiente & 100 & -- & 100 & -- \\
\hline $\begin{array}{l}\text { La metodología me ayudó a trabajar } \\
\text { colaborativamente }\end{array}$ & 100 & -- & 100 & -- \\
\hline $\begin{array}{l}\text { La metodología favoreció mi capacidad de análisis } \\
\text { La metodología fomentó mi habilidad para resolver } \\
\text { problemas e identificar elementos relevantes }\end{array}$ & 100 & -- & 94 & $\mathrm{~N} / \mathrm{C}$ \\
\hline $\begin{array}{l}\text { La metodología me motivó a asumir una mayor } \\
\text { responsabilidad en mi propio aprendizaje }\end{array}$ & 100 & -- & 100 & -- \\
\hline
\end{tabular}

Fuente: Elaboración propia (2018)

n 2015: 11 estudiantes - n 2016: 16 estudiantes.

N/C: No contesta

Respecto a la dinámica que las/os estudiantes experimentaron durante la sesión, sólo una persona manifestó que el trabajar en equipo no facilitó el análisis de la temática, al igual que el hecho de utilizar el material bibliográfico durante las sesiones no le motivó a estudiar y profundizar en la temática, por lo cual no consideraba que la metodología tuviera algún efecto en ella/él. Sin embargo el $100 \%$ de los participantes, creen que los casos que se revisaron durante las sesiones de trabajo, el análisis que de allí surgió y por lo tanto el conocimiento adquirido, pueden ser útiles para aplicar en la vida diaria.

Tabla 2. Evaluación de la dinámica de trabajo

\begin{tabular}{|l|c|c|c|c|}
\hline \multicolumn{1}{|c|}{ Aspecto que el/la estudiante evalúa } & Si & No & Si & No \\
\hline $\begin{array}{l}\text { Considero que trabajar en equipo facilitó el análisis, } \\
\text { la resolución del problema e identificación de } \\
\text { elementos relevantes }\end{array}$ & 91 & 9 & 100 & -- \\
\hline $\begin{array}{l}\text { El utilizar artículos de divulgación, libros u otro } \\
\text { material bibliográfico relacionado, me motivó a } \\
\text { estudiar la temática del módulo }\end{array}$ & $82^{*}$ & 9 & $94^{*}$ & N/C \\
\hline $\begin{array}{l}\text { Considero que los casos revisados me ayudarán a } \\
\text { aplicar los conocimientos a la vida diaria }\end{array}$ & 100 & -- & 100 & -- \\
\hline
\end{tabular}

Fuente: Elaboración propia (2018)

n 2015: 11 estudiantes $-\mathrm{n}$ 2016: 16 estudiantes

N/C: No contesta

Al ser consultados sobre si son conscientes de haber adquirido algunos elementos para su aprendizaje durante las sesiones, un $25 \%$ de los estudiantes reconocen que en un principio 
fue dificil identificar los elementos que se necesitaban para desarrollar la temática. En efecto, en ambas experiencias se pudo detectar cierto nivel de inseguridad y desorientación que se minimizaba cuando compartían con el resto de los integrantes del grupo algunas ideas y abordaban en conjunto los principales hallazgos. Otro aspecto evaluado, fue el tiempo destinado a las sesiones en el cual un $87 \%$ de las/os estudiantes del segundo grupo considera que el trabajo en dos sesiones fue insuficiente y que necesitaban un poco mas de tiempo para poder profundizar en la temática, en contraste con el $9 \%$ del primer grupo.

Tabla 3. Evaluación de aprendizaje asimilado

\begin{tabular}{|l|c|c|c|c|}
\hline \multicolumn{1}{|c|}{ Aspecto que el/la estudiante evalúa } & $\mathbf{2 0 1 5}(\mathbf{\%})$ & $\mathbf{2 0 1 6}(\mathbf{\%})$ \\
\hline $\begin{array}{l}\text { Me fue fácil identificar el problema y los elementos a } \\
\text { estudiar }\end{array}$ & 100 & -- & $69 *$ & 25 \\
\hline $\begin{array}{l}\text { El tiempo destinado a la discusión y resolución fue } \\
\text { suficiente }\end{array}$ & 91 & 9 & 13 & 87 \\
\hline $\begin{array}{l}\text { La guia del tutor favoreció el trabajo de mi equipo } \\
\text { Aprendi sobre los temas analizados por mi equipo }\end{array}$ & 100 & -- & 100 & -- \\
\hline Aprendi sobre los temas analizados por el otro equipo & 91 & -- & 100 & -- \\
\hline
\end{tabular}

Fuente: Elaboración propia (2018)

n 2015: 11 estudiantes - n 2016: 16 estudiantes

Respecto a los instrumentos de evaluación utilizados en ambos grupos, gran parte de los estudiantes considera apropiados los items con los cuales se les califica, al igual que la instancia y el instrumento con el cual se autoevaluan y co-evaluan a su compañero. Aún cuando esta es una metodología que se aleja de las formas tradicionales de evaluación, un $27 \%$ de quienes cursaron el módulo prefieren ser calificados con un certamen y las clases expositivas, argumentando que no les gusta trabajar en equipo, siendo esta la principal razón por la cual no se sienten comodos con esta metodología.

Tabla 4. Instrumentos de evaluación

\begin{tabular}{|l|c|c|c|c|}
\hline Aspecto que el/la estudiante evalúa & \multicolumn{2}{c|}{$\mathbf{2 0 1 5}(\%)$} & \multicolumn{2}{c|}{$2016(\%)$} \\
\hline $\begin{array}{l}\text { La escala de estimación conceptual me parece } \\
\text { adecuada para evaluar la actividad }\end{array}$ & 100 & -- & $88^{*}$ & 6 \\
\hline $\begin{array}{l}\text { Considero apropiado autoevaluarme y evaluar a } \\
\text { mis compañeros }\end{array}$ & 91 & 9 & 81 & 19 \\
\hline Prefiero que me evaluen con un certamen & 27 & 73 & -- & $88^{*}$ \\
\hline
\end{tabular}

Fuente: Elaboración propia (2018)

n 2015: 11 estudiantes - n 2016: 16 estudiantes

Por último, al ser consultados sobre la evaluación general de la actividad a la generalidad de las/os estudiantes le gustó utilizar esta metodología, y un $91 \%$ considera que no requiere 
El Aprendizaje Basado en Problemas (ABP) en Ciencias Sociales: resultados de una experiencia en estudiantes de postgrado en Chile

mucho tiempo y su rendimiento no se ve perjudicado por la misma (frente a un $9 \%$ manifiesta que sí) en el primer grupo. Aún cuando consideran que es una buena metodología de trabajo un $50 \%$ la prefiere por sobre las clases magistrales en el segundo grupo, frente al 91\% que prefiere esta metodología por sobre las clases traidicionales del primer grupo. Al ser consultados al respecto, manifestaban que les gustaría que algunos módulos fueran impartidos de esta forma pero no toda la asignatura. Esto se condice con lo que finalmente plantean, respecto a si les gustaría utilizar esta metodología en otras asignaturas.

Tabla 5. Evaluación general de la actividad

\begin{tabular}{|c|c|c|c|c|}
\hline \multirow[t]{2}{*}{ Aspecto que el/la estudiante evalúa } & \multicolumn{2}{|c|}{$2015(\%)$} & \multicolumn{2}{|c|}{$2016(\%)$} \\
\hline & Si & No & Si & No \\
\hline $\begin{array}{l}\text { Me gustó utilizar el Aprendizaje Basado en } \\
\text { Problemas }\end{array}$ & 100 & -- & $94 *$ & -- \\
\hline $\begin{array}{l}\text { La metodología de trabajo requiere mucho tiempo y } \\
\text { perjudica mi rendimiento }\end{array}$ & 9 & 91 & -- & 100 \\
\hline Prefiero ABP por sobre clases magistrales & 91 & 9 & $50 *$ & 31 \\
\hline $\begin{array}{l}\text { Me gustaria utlizar más frecuentemente esta } \\
\text { metodología en esta y otras asignaturas }\end{array}$ & 100 & -- & 81 & 19 \\
\hline
\end{tabular}

Fuente: Elaboración propia (2018)

n 2015: 11 estudiantes - n 2016: 16 estudiantes

Además de evaluar los aspectos anteriormente mencionados, las/os estudiantes tenían la posibilidad de realizar comentarios de forma voluntaria sobre la metodología utilizada. En los aspectos a mejorar sugieren agregar o utilizar material audiovisual durante la clase, aportar con un mayor número de material bibliográfico durante el desarrollo de la sesión considerando que el tiempo para el estudio independiente es muy breve entre una sesión y otra o bien, realizar la clase en el laboratorio de computación.

Entre los aspectos positivos que las/os estudiantes destacan del ABP, es que aun cuando en un principio se encuentran en un momento de incertidumbre frente a aquello que deben encontrar, esta presión genera y estimula un aprendizaje sobre el tema y motiva el trabajo independiente en casa, situación que no suele darse en todas las asignaturas. Consideran que es una metodología dinámica y enriquecedora que fomenta la colaboración y el trabajo en equipo por lo cual debiera replicarse en otras instancias. Por último, frente al rol del docente que ejerce una labor de guía durante las sesiones consideran que es vital ya que permite que el grupo no se desvie del tema central.

Desde el punto de vista docente, se aprecia que la metodología intenciona que los estudiantes generen un debate y diálogo constante con sus compañeras/os y por lo tanto compartan nuevas ideas que los ayude a analizar y resolver el problema seleccionado facilitando la búsqueda de nuevos hallazgos. Es una metodología que intenciona la escucha 
activa y la elaboración de ideas sobre la base de lo que se comparte con el resto del equipo, y los ayuda a fortalecer aspectos como la empatía y el respeto por la opinión de los demás, asi como la asertividad para comunicar sus ideas sin descalificar las otras opiniones.

\section{Discusión}

La Universidad de Concepción recibe cada año miles de estudiantes presentando cada uno de ellos una diversa condición socioeconómica y procedencia en cuanto a la educación recibida en los años previos a su entrada la Universidad. Esto deja en evidencia una situacion de desigualdad entre las/os estudiantes en cuanto a habilidades y competencias clave para enfrentar su futuro desempeño laboral. Esta técnica si bien no pretende (y no puede) acabar con ese nivel de desigualdad - tan solo plantearselo sería imposible considerando los aspectos que rodean este concepto - si pretende generar y desarrollar habilidades o competencias independiente del nivel socioeconómico de sus estudiantes o de algunas dificultades personales que pudieran presentar.

En este sentido con la metodología $\mathrm{ABP}$ se puede apuntar a que los/as estudiantes se encuentren al mismo nivel de trabajo, que compartan responsabilidades en cuanto al conocimiento que deben adquirir, que desarrollen la capacidad de autocrítica y de evaluar responsablemente la labor de quienes integran su equipo.

Bajo esta premisa, resulta casi obvio asumir que la clásica modalidad de enseñanza, aquella focalizada en los contenidos, con técnicas de evaluacion que se limitan a comprobar la memorizacion de información y hechos, no representa un desafío al estudiante a alcanzar niveles cognitivos mas altos. El profesor transmite y el estudiante es un receptor pasivo. El mundo en el cual estamos insertos, demanda profesionales que puedan adaptarse a las distintas contingencias, que tengan capacidad de trabajo en equipo en la resolución de problemas, capacidad de análisis y un efectiva comunicación. Bajo estas demandas, surge la necesidad de preparar a las/os estudiantes para que puedan superar exitosamente diversas dificultades propias de la realidad y para los cuales las modalidades tradicionales de formación ya no son efectivas. En este contexto, la metodología de ABP constituye una respuesta importante en la formación de profesionales competentes. Esta empodera y responsabiliza a los estudiantes en cuanto a la gestión del conocimiento pero tambien representa un desafío para los docentes en cuanto significa una modernizacion de sus técnicas de enseñanza y una constante re evaluación de las mismas, ya que implica relegar su rol protagónico a un rol de facilitador y de apoyo.

Considerando los resultados obtenidos con esta metodología y expuestos anteriormente, se demuestra que la técnica promueve el aprendizaje autónomo gracias al rol que ejerce el 
El Aprendizaje Basado en Problemas (ABP) en Ciencias Sociales: resultados de una experiencia en estudiantes de postgrado en Chile

profesor guía quien delega en el estudiante la responsabilidad por buscar aquello que desea aprender, siendo este elemento el objetivo principal del ABP.

Respecto a la dinámica que se experimentaba en cada sesión, esta difiere según los períodos. Por lo cual cabria preguntarse si esta diferencia significativa de opiniones puede radicar en las caracterísiticas individuales de cada estudiante, en la dinámica que haya presentado cada uno de los grupos o bien en el nivel de dificultad que haya generado la noticia con la cual trabajó cada uno de ellos y que difería cada uno de los periodos, lo cual puede haber facilitado o dificultado la tarea de encontrar los principales elementos que se necesitaba profundizar. Este puede ser un aspecto relevante a considerar y estudiar en el futuro, ya que el tiempo que se dedica a la sesión, al menos en el Diplomado ( 8 horas), no es posible de modificar.

Al respecto, el factor tiempo fue otro de los items evaluados que generó mayor discusión en cada una de las sesiones que se desarrolló el Diplomado (ambas versiones). Esto, debido que al escaso tiempo con el cual se contaba los estudiantes sugerían trabajar en un laboratorio de computación con el fin de acceder de forma mas rapida y expedita a fuentes de información. Si bien es cierto esto es una buena idea, el trabajar en un entorno que no facilite la comunicación entre el equipo hace que la técnica pierda el sentido por lo cual puede evaluarse esta sugerencia pero adaptarla a la realidad de la metodología y de las características del módulo en el cual se aplica.

Finalmente y sobre la base de lo ya expuesto, el ABP es una metodología que además de generar y promover la autonomia en la busqueda de información y con ello su aprendizaje, permite desarrollar ciertas habilidades en los estudiantes que los ayudara a enfrentar las diversas situaciones con las cuales tendrán que lidiar una vez egresen de la universidad, siendo por tanto una herramienta que debiera ser utilizada en los estudiantes de pregrado de nuestras casas de estudio. Actualmente, en la carrera de Trabajo Social de la Universidad de Concepción se está llevando a cabo una asignatura denominada Aproximación a la Intervención Social y la cual se esta trabjando a través del método de tutor desde el año 2016, compartiendo características muy similares al ABP. Este 2018 se llevará a cabo su tercera versión y se prevé la realización de una sistematización de la misma al final del presente año.

\section{Agradecimientos}

Agradecimientos a la Dra. Carola Bruna, Docente de la Universidad de Concepción quien facilitó los instrumentos de evaluación para trabajo colaborativo y percepción del estudiante utilizados en ambas experiencias cuyo resultados fueron presentados en el artículo. 
This article was elaborated in the context of INCASI Network, a European project that has received funding from the European Union's Horizon 2020 research and innovation programme under the Marie Skłodowska-Curie GA No 691004 and coordinated by Dr. Pedro López-Roldán". "This article reflects only the author's view and the Agency is not responsible for any use that may be made of the information it contains".

\section{Referencias}

Barber, M., \& Mourshed, M. (2008). Cómo hicieron los sistemas educativos con mejor desempeño del mundo para alcanzar sus objetivos. Santiago: McKinsey \& Company.

Barrel, J. (1999). Aprendizaje Basado en Problemas, un enfoque investigativo. Buenos Aires: Editorial Manantial.

Barrows, H. (1986). A taxonomy of problem-based learning methods. Medical Education , $20,481-486$.

Barrows, H. (1996). Problem-Based learning in medicine and beyond: a brief overview. En L. Wilkerson, \& W. Gijselaers, Bringing Problem-Based Learning to Higher Education: Theory and Practice (págs. 3-12). San Francisco: Jossey-Bass Publishers.

Fernández, L., \& Zarauza, D. (2011). Jovenes universitarios con estudios de postgrado. Perspectivas en Argentina: trayectorias educativas y laborales de estudiantes de los posgrados de las Facultad de Bellas Artes de la UNLP . XI Coloquio Internacional sobre gestión universitaria en America del Sur (págs. 1-13). Florianopolis: II Congreso Internacional IGLU.

Laudadío, J., \& Mazzitelli, C. (2015). Estilos de enseñanza de los docentes de distintas carreras de nivel superior vinculadas con las ciencias naturales. Revista Educación , 14 (46), 9-25.

Morales, P., \& Landa, V. (2004). Aprendizaje Basado en Problemas . Theoría , 13, 145 157.

Morales, P. (2009). Logros en motivación y el tercer nivel de estructura del conocimiento: un estudio empírico en contextos de aprendizaje correspondientes a una modalidad híbrida ABP. Revista Educación , 18 (34), 73-92.

Programa de Estudios sobre la Responsabilidad Social . (2013). Modelo de Enseñanza de Competencias Genéricas. Programa Competencias Genéricas Convenio de Desempeño UCO1204. Concepción: Universidad de Concepción. 
El Aprendizaje Basado en Problemas (ABP) en Ciencias Sociales: resultados de una experiencia en estudiantes de postgrado en Chile

Rivera, Bruna, Reinicke, Grez \& Santamaría (2014) Experiencia de trabajo colaborativo en la confección de una rúbrica analítica para auto y coevaluación de trabajo colabora-tivo. (Resumen de Congreso) Revista Educación en Ciencias de la Salud, 11 (1), 92.

Rodriguez, R., Hernández, N., \& Diaz, M. (2007). Cómo planificar asignaturas para el aprendizaje de competencias. Universidad de Oviedo. Oviedo: Documentos ICE Universidad de Oviedo.

Solís, P., \& Blanco, E. (2014). La desigualdad en las trayectorias educativas y laborales de los jóvenes de la ciudad de México: un panorama general. En E. Blanco, P. Solís, \& H. Robles, Caminos desiguales. Trayectorias educativas y laborales de los jovenes en la ciudad de México (págs. 21-37). México D.F: Instituto Nacional para la evaluación de la educación.

Vargas, J., Chiroque, E., \& Vega, M. (2016). Innovación en la docencia universitaria. Una propuesta de trabajo multidisciplinario y colaborativo en educación superior. Revista Educación , 15 (48), 67-84 